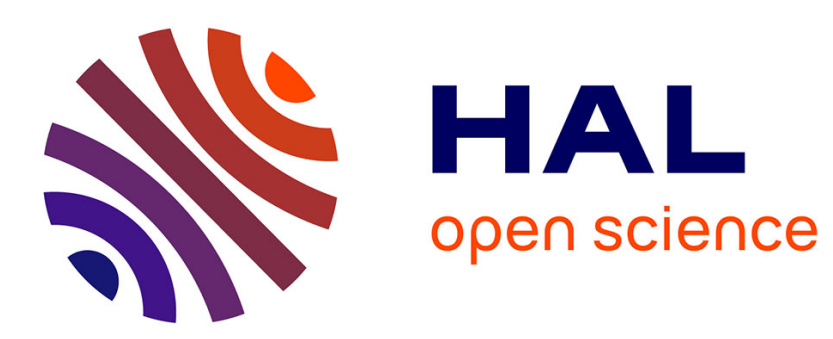

\title{
Investigation of Single Event Effects in a Resistive RAM memory array by SPICE level simulation
}

\author{
K. Coulié, Hassen Aziza, W Rahajandraibe
}

\section{To cite this version:}

K. Coulié, Hassen Aziza, W Rahajandraibe. Investigation of Single Event Effects in a Resistive RAM memory array by SPICE level simulation. 2021 IEEE 22nd Latin American Test Symposium (LATS), Oct 2021, Punta del Este, Uruguay. 10.1109/LATS53581.2021.9651871 . hal-03512542

\section{HAL Id: hal-03512542 \\ https://hal.science/hal-03512542}

Submitted on 5 Jan 2022

HAL is a multi-disciplinary open access archive for the deposit and dissemination of scientific research documents, whether they are published or not. The documents may come from teaching and research institutions in France or abroad, or from public or private research centers.
L'archive ouverte pluridisciplinaire HAL, est destinée au dépôt et à la diffusion de documents scientifiques de niveau recherche, publiés ou non, émanant des établissements d'enseignement et de recherche français ou étrangers, des laboratoires publics ou privés. 


\title{
Investigation of Single Event Effects in a Resistive RAM memory array by SPICE level simulation
}

\author{
K. Coulié, H. Aziza, W. Rahajandraibe \\ IM2NP-UMR CNRS 6242 / Aix-Marseille University \\ IM2NP, IMT Technopôle de Château - Gombert, 13451 Marseille Cedex 20, France \\ e-mail: hassen.aziza@im2np.fr, wenceslas.rahajandraibe@im2np.fr, karine.coulie@im2np.fr
}

\begin{abstract}
Emerging non-volatile memories, based on resistive switching mechanisms and known as Resistive Random Access Memory (RRAM), are attractive candidates to overcome power, cost and integration density limitations of conventional memories. Moreover, RRAM has exhibited very good tolerance to radiation. In this context, this paper proposes to investigate Single Event Effects in RRAM memory arrays. The decoding circuitry of the memory array, including bit line and source line drivers is targeted. Currents generated by an ionizing particle crossing the memory array are first injected at specific nodes of the memory circuit. Their impact is evaluated by extracting the resistance state of each cell of the memory array before and after the ionizing particle strike. Worst cases scenarios are studied in order to point out the most sensitive configurations able to induce SEE.
\end{abstract}

Keywords - OxRAM, RRAM, SEE, SPICE simulation.

\section{INTRODUCTION}

Among the most promising technologies to meet the ITRS requirements for non-volatile memories, Resistive RAM (RRAM) [1-4] are attractive candidates, due to their appealing performances. Indeed, compared to conventional floating gate technologies, they gather fast write/read operations, low power consumption, CMOS compatibility and high endurance. It is worth noting that in literature, the acronym RRAM is often used to refer to both Oxide Random Access Memory (OxRAM) and Conductive-Bridging Random Access Memory (CBRAM). In the rest of the paper, OxRAM technology will be considered. As radiation effects are nowadays recognized as a real reliability concern, it is mandatory to evaluate the hardness of these particular devices against radiations. Studies exist about the specific radiation constraint [5-9], but available works often address dose effects in the resistive switching element. Alternatively, RRAMs elementary cells based on hafnium oxide have demonstrated a tolerance against various kinds of radiations [10-15]. In OxRAM memories, MIM (Metal-Insulator-Metal) structure is used as a single-bit memory cell, while the semiconductor transistor subsystem enables performing all the peripheral operations (input/output, coding/decoding, line driving, and sense amplification). In the complementary study presented in this paper, as in previous investigations [16,17], the case of an ion crossing the MIM (Metal-Insulator-Metal) structure of an OxRAM cell is not considered. However, considering the insulator thickness and the probability of occurrence of such an event, we can expect that the impact on the global SER should be low if only SEE are considered (no combined effects with dose).

The aim of the paper is to perform a complementary analysis of the ones presented in [16,17], which focus on Single Event Effects (SEE) in the close proximity of the OxRAM cell (i.e., selection transistors). In these papers, the studied OxRAM cell has shown low sensitivity to SEE. A threshold effect was pointed out and quantified in terms of current features. It was shown that the only configurations inducing effects were simultaneous impacts in a NMOS and a PMOS transistors, one being connected to the Bottom Electrode (BE) node and the other to the Top Electrode (TE) node of the OxRAM cell. Then it was observed that the sensitive NMOS should be physically close to the sensitive PMOS to induce SEE occurrence. The occurrence of such a case would probably be low but possible. Indeed, depending on the considered technology and on the memory array, the distance between two sensitive transistors can vary from tens to hundreds of microns. So, only a particle crossing the structure (i.e., a particle with a long range), could impact the OxRAM memory array behavior. Note that the simulations were performed for a specific structure corresponding to the worst case for single event radiation effects. However, in order to improve integration density and scalability in emerging technologies, 3D stacking technology is adopted where NMOS and PMOS transistors could be physically close. In that case, the OxRAM technology sensitivity to SEE could increase. The remainder of this paper is organized as follows. Section

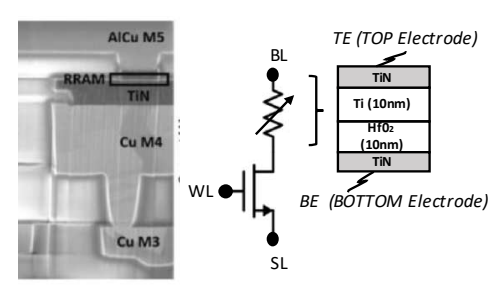

(a)

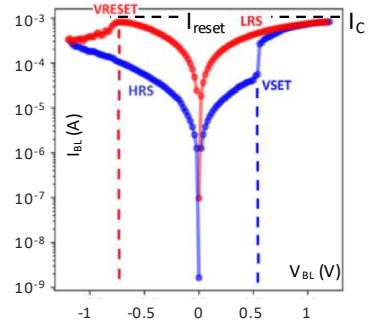

(c)
Fig. 1. (a) TEM cross-section of an OxRAM device (b) symbol view of a 1T-1R cell (c) OxRAM I-V characteristic in log scale. 


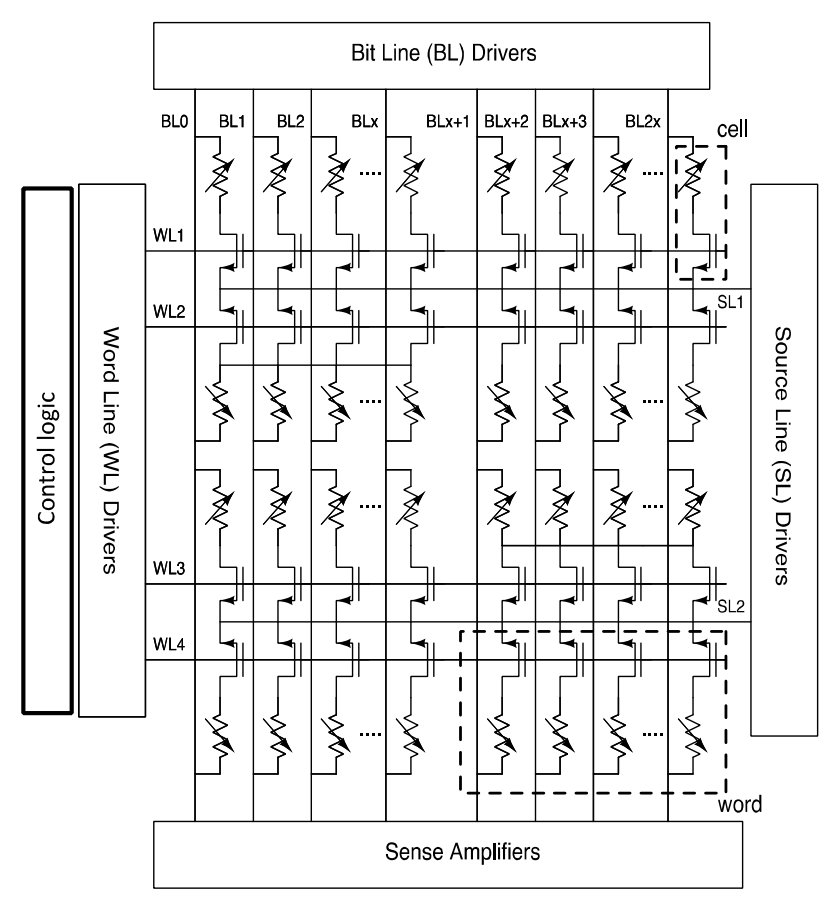

Fig. 2. Elementary memory array architecture used for simulations

II presents the OxRAM technology. In Section III, SEE is analyzed at the memory array level. Section IV presents simulation results showing the effect of SEE. Finally, Section V concludes this paper.

\section{RRAM TECHNOLOGY}

An OxRAM memory cell consists of two metallic electrodes that sandwich a thin dielectric layer serving as a permanent storage medium. Its resistance may be switched between ' 0 ' and ' 1 ' states. The OxRAM Metal-Insulator-Metal (MIM) structure can be easily integrated in the Back-End Of Line (BEOL) on top of the CMOS subsystem [6-10]. Oxide-based resistive random access memory (so-called OxRAM) elements present a lot of interesting features like high integration density, high-speed operations (write/erase/read) and satisfactory reliability performances in terms of retention and cycling. Fig. $1 \mathrm{~b}$ shows the basic 1T-1R memory cell, where one MOS transistor is connected in series with an OxRAM cell. Fig. 1c presents a typical 1T-1R OxRAM I-V characteristic in logarithmic scale. Based on the I-V curve, the memory cell operation can be seen as follows: after an initial electro-FORMING (FMG) step [18], the memory element can be reversibly switched between the Low Resistance State (LRS) and the High Resistance State (HRS). Resistive switching corresponds to an abrupt change between the HRS and the LRS. The resistance change is triggered by applying specific biases across the $1 \mathrm{~T}-1 \mathrm{R}$ cell, i.e., $\mathrm{V}_{\mathrm{SET}}$ to switch to LRS and $V_{R S T}$ to switch to HRS. In the 1T-1R configuration, the transistor controls the amount of current flowing through the cell according to its gate voltage bias. The maximum current allowed by the select transistor is called the compliance current and is referred to as $\mathrm{I}_{\mathrm{C}}$ in Fig. 1c. $\mathrm{I}_{\mathrm{C}}$ controls the

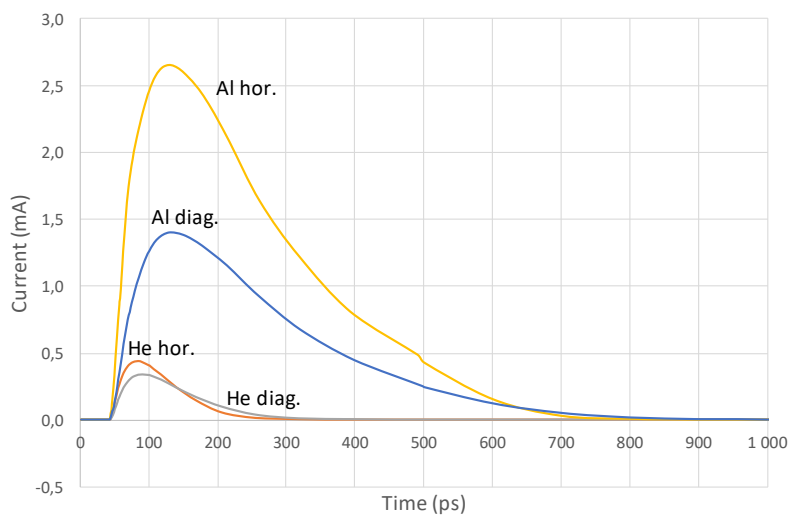

Fig. 3. Currents generated by Alpha and Aluminum particles in two different configurations: horizontal (hor.) and diagonal (diag.).

LRS resistance value in the SET state as well as the maximal RESET (RST) current $\mathrm{I}_{\text {reset. }}$

\section{SEE IN THE OXRAM MEMORY ARRAY}

Fig. 2 presents the OxRAM memory array, studied in this work, which is composed of a word line driver to select the active row $\left(\mathrm{WL}_{\mathrm{X}}\right)$, a bit line driver to select the active bit lines $\left(B L_{X}\right)$ during a FORM/SET and READ operations, and a source line driver to select a specific source line $\left(\mathrm{SL}_{\mathrm{X}}\right)$ during RST. Sense amplifiers are used to convert the cell current into a logical value at the circuit output during a READ operation. Memory cells are usually grouped into 8,16 or 32 bits to form a memory word (dashed line). During the READ operations, the sense amplifier compares the cell current to a strictly controlled reference current.

The whole structure is simulated using Eldo simulator at transistor level (SPICE simulation). The effect of a particle crossing the structure is simulated by adding a parasitic current source at the impacted node(s). The current value is negative when a NMOS transistor is struck and positive when a PMOS transistor is struck. The current source comes from the realistic simulation of a particle crossing a silicon volume using TCAD device simulation tools (Synopsis (C). The effect of the ion strike is simulated using the Heavy Ion module of Synopsis [19], considering an electron-hole pair column centered on the ion track axis. The Linear Energy Transfer is defined as the energy lost by the particle, by unit of length and varies along the track depending on the initial energy of the particles [20]. An actual variation of the LET was integrated in our simulations, based on the value given by SRIM tables [21]. Two kinds of ions are simulated: Alpha and Aluminum ions. The simulated Alpha particle is supposed to cross the device with an initial energy of $0.6 \mathrm{MeV}$, which corresponds to a range of $2.3 \mu \mathrm{m}$ in the silicon volume. It corresponds to the end of an Alpha particle path of $1.47 \mathrm{MeV}$, generated by the initial interaction of a thermal neutron with boron-10 (Alpha contamination). The case of a more energetic particle is also considered. It is a $50 \mathrm{MeV}$ Aluminum which could be produced by the interaction of fast protons with silicon [22]. Two geometries are simulated for the two ions: crossing horizontally 


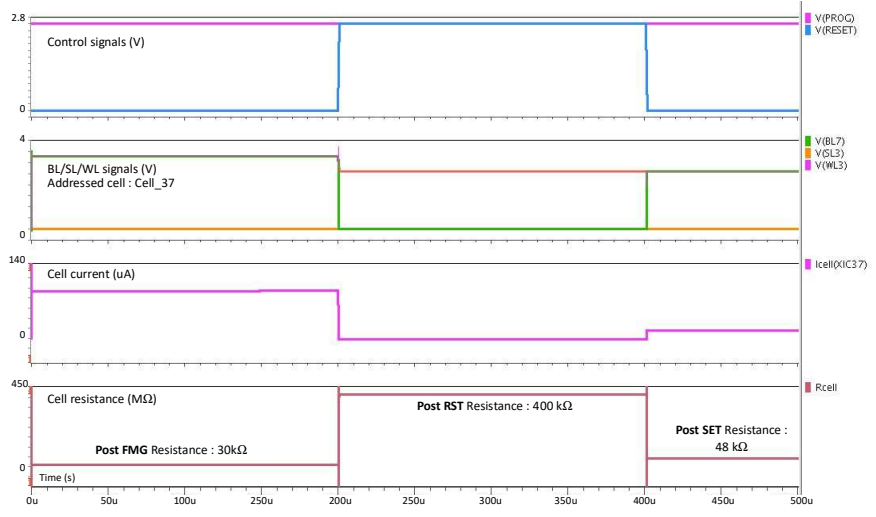

Fig. 4. Transient simulation results after FMG, RST and SET operations.

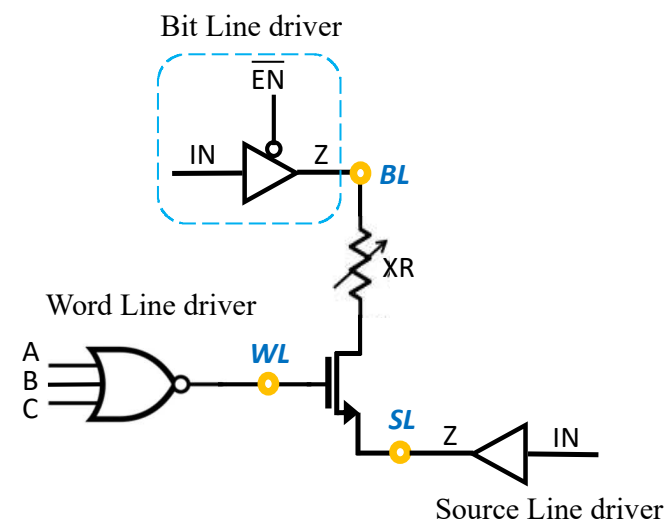

Fig. 5. Schematic diagram of one memory point and its peripheral circuit.

(hor.) or diagonally (diag.) the silicon volume. The associated currents are presented in Fig. 3. Al hor. corresponds to a total deposited charge of $723 \mathrm{fC}, \mathrm{Al}$ diag. to $398 \mathrm{fC}$, He hor. to $44 \mathrm{fC}$ and $\mathrm{He}$ diag. to $43 \mathrm{fC}$.

Thus, SEE is evaluated by injecting and simulating sequentially parasitic current source using the electrical simulator. Current sources, reflecting SEE, are injected at specific nodes of the memory array presented in Fig. 2. The goal is to determine which current source causes logical failures and which one does not but causes an important shift of OxRAM resistances ("analog" faults). To evaluate the impact of each source on the memory circuit, the simulation process is automated. Currents are injected sequentially and the circuit responses are automatically extracted.

As the aim of this study is to get information about the worstcase configuration, the configurations able to induce a change in the initial state will be the only ones studied here. The investigation focuses on the most sensitive peripheral circuits to demonstrate the impact of a particle strike on the steady-state of the transistors directly involved to program the RRAM value. Among possible states (RST, SET, READ, WRITE, STBY), the standby operation is the longer state in terms of duration. Then the probability for a radiation event to occur during standby is the highest and this configuration is particularly explored here.

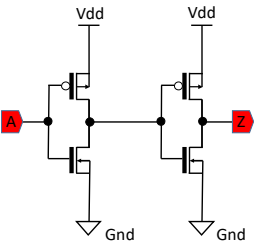

(a) WL driver

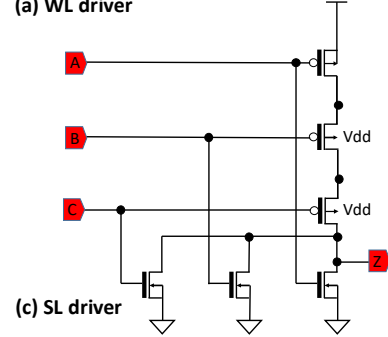

Figure 6. Sensitive node location at the memory array driver level

\section{EFFECT OF SEE IN THE OXRRAM ARRAY}

\section{1) Fault simulation strategy}

We implemented the memory circuit presented in Fig. 2 using a $0.13 \mu \mathrm{m}$ High Voltage CMOS technology offering a $3.3 \mathrm{~V}$ supply voltage. This technology was targeted because both, measurements and model calibration, were available for the 130 $\mathrm{nm}$ technology. Moreover, transistors withstanding high voltages are available in the $130 \mathrm{~nm}$ technology. The latter are required during the FORMING operation achieved at 3.3V. To verify the operation of our design scheme, SPICE simulations are performed using the Eldo simulator. Various methodologies have been developed since several years to get reliable results about SEE sensitivity using SPICE simulations [23-27]. Using this kind of simulation decreases considerably the simulation time. Indeed, SPICE simulations run at least 100 times quicker than TCAD simulations. It allows a very quick analysis even if the accuracy is limited for the study of radiations effects [2829]. It is particularly convenient when mixed-mode simulation is used to simulate the peripheral circuits at SPICE level and the impacted cell at TCAD level [30].

Table I presents typical voltage levels applied to the memory cells during FMG, RESET, SET, READ and STBY operations. These voltage levels are provided by the Bit Line driver s (BL), the Source Line drivers (SL) and the Word Line (WL) drivers of Fig. 4.

TABLE I. OPERATING VOLTAGE LEVELS (H:2.5V, HV:3.3V, L:0V)

\begin{tabular}{cccccc}
\hline \hline & FMG & RST & SET & READ & STBY \\
\hline BL & HV & L & H & HI & HI \\
SL & L & H & H & H & L \\
WL & H & H & H & H & H/L \\
\hline \hline
\end{tabular}




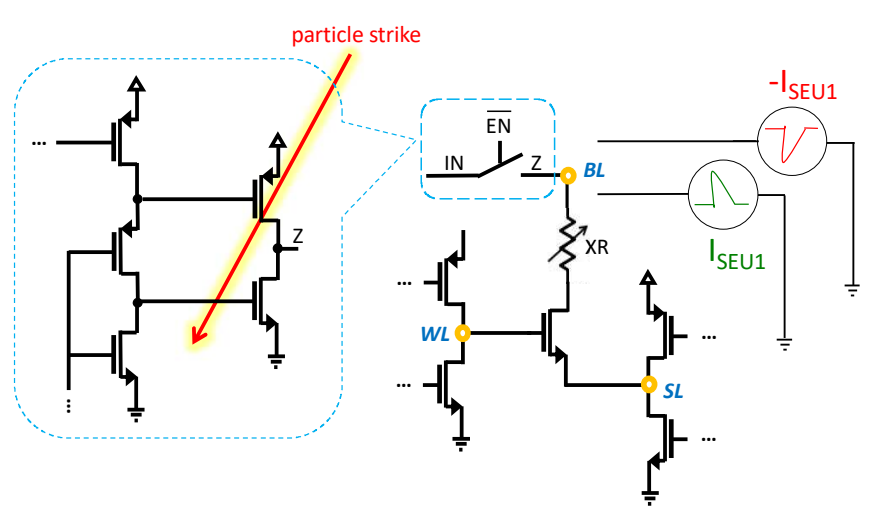

Fig. 7. Schematic diagram of one memory cell and its peripheral circuit at the transistor level.

Transient simulation results are presented in Fig. 4 considering the voltage levels presented in Table I. One cell of the memory is addressed (Cell 37) by activating WL3, BL7 and SL3. The cell current $\mathrm{I}_{\text {cell }}(\mathrm{XIC} 37)$ and the final cell resistance $\left(\mathrm{R}_{\text {cell }}\right)$ are extracted after FMG $(\sim 30 \mathrm{k} \Omega)$, RST $(\sim 30 \mathrm{k} \Omega)$ and SET $(\sim 45$ $\mathrm{k} \Omega$ ) operations. In this study, the investigation is focused on the peripheral circuit of one memory point (XR) depicted in Fig. 5. As shown, three logic gates are involved in the memory configuration, namely the bit line (BL), the word line (WL) and the source line (SL) drivers.

In order to better understand the electrical mechanism managing the bias voltage of each terminal (BL, WL and SL), the transistor level schematic of each CMOS driver is depicted in Figs 6 . The schematic diagram of one memory point is presented in Fig. 7 where only the transistors directly connected to the memory nodes are shown. As we can state, each terminal (BL, WL and SL) are faced with PMOS and NMOS transistors that are used to set the good operating voltage, according to the chosen configuration, as summarized in Table I.

Particle strike is supposed to cause a transient error on the nominal value of the bias voltage at each node (BL, WL and SL) directly connected to the RRAM memory. These specific nodes are considered as sensitive nodes regarding fault simulation due to particle crossing. Current spikes resulting of particle strikes are injected at these sensitive nodes and the state of each memory cell of the array is evaluated. Based on the considered memory array architecture, the only operating stage subject to

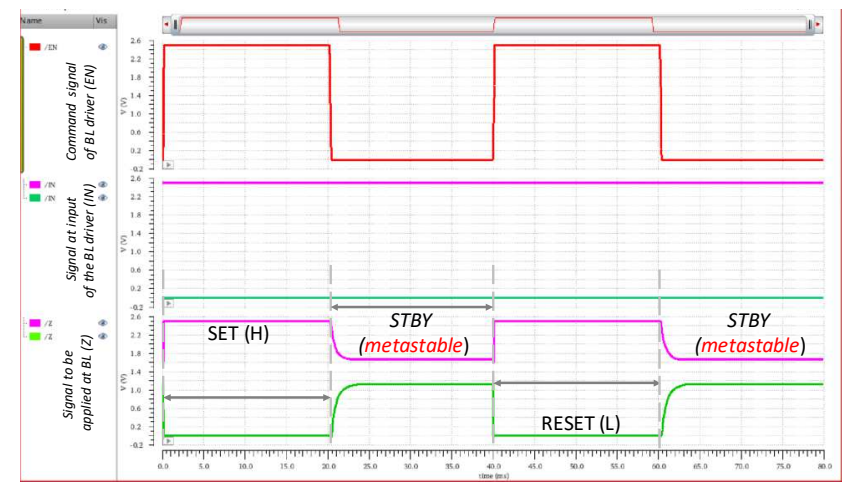

Fig. 8: Electrical value simulated at the output of the BL driver (BL node)

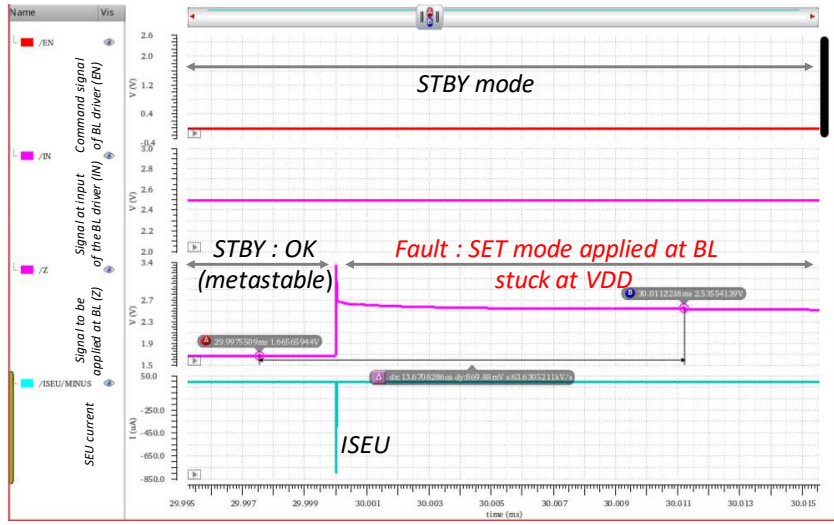

Fig. 9: Simulation of SEU strike on the BL driver (passgate).

SEE errors, leading to resistance drift is the STBY stage (Table II). In this configuration, the electrical values of the RRAM terminals (BL, SL and WL) are respectively high impedance (HI), low (L) and high or Low $(\mathrm{H} / \mathrm{L})$. To well understand the STBY configuration, we simulated the electrical operation of each driver (Fig. 8) focusing on the BL driver with HI value (depicted as a controlled switch in the Fig. 5). The BL driver is in fact a CMOS tri-state gate. When the EN terminal is set, the gate is on and the output voltage at the terminal $\mathrm{Z}(\mathrm{BL})$ is the same as the input one (IN). From an electrical point of view, these values correspond to $\mathrm{VDD}=2.5 \mathrm{~V}(\mathrm{H})$ or $\mathrm{GND}=0 \mathrm{~V}(\mathrm{~L})$. However, when the gate is off, the voltage value at $\mathrm{Z}$ terminal is around $\mathrm{VDD} / 2$ depending on the voltage value at the input. This value corresponds to a metastable state (HI). This configuration is associated with the STBY state. Fig. 9. shows the impact of a particle striking the peripheral transistor $\mathrm{N}_{\mathrm{BL}}$ of Fig 6 , during the STBY mode. This is done by injecting a very short current pic $\left(\mathrm{I}_{\mathrm{SEU}}\right)$ at the $\mathrm{Z}$ node of the BL driver. We can see in Fig. 9 that the voltage value at node $\mathrm{Z}$ switches to VDD (stuck at VDD) leading to a fault on the memory configuration.

Several scenarios are possible depending on the path and the energy of the particle. We will focus in this paper only on the particles striking the peripheral transistors $\left(\mathrm{P}_{\mathrm{BL}} ; \mathrm{N}_{\mathrm{BL}}\right),\left(\mathrm{P}_{\mathrm{WL}}\right.$; $\left.\mathrm{N}_{\mathrm{WL}}\right)$ and $\left(\mathrm{P}_{\mathrm{SL}} ; \mathrm{N}_{\mathrm{SL}}\right)$ of Fig 7 . Indeed, these transistors act directly on the potential difference between the memory terminals. The next section are dedicated to the worst case scenario identification.

TABLE II. PARTICLE STRIKE IMPACT

\begin{tabular}{cccccc}
\hline \hline & FMG & RST & SET & READ & STBY \\
\hline Impact & NO & NO & NO & NO & YES \\
\hline \hline
\end{tabular}

\section{2) Worst cases scenarios simulation}

The worst-case scenarios are related to a particle strike impacting both the Bit Line voltages and Source Line voltages in STBY mode. Thus, extensive simulations are performed to assess the robustness of the memory array versus particles striking at these specific locations. Two configurations are studied. The first one considers a memory cell at LRS (Low Resistance State: $48 \mathrm{k} \Omega$ ). 
The second one considers a memory cell at HRS (High Resistance State: $400 \mathrm{k} \Omega$ ).

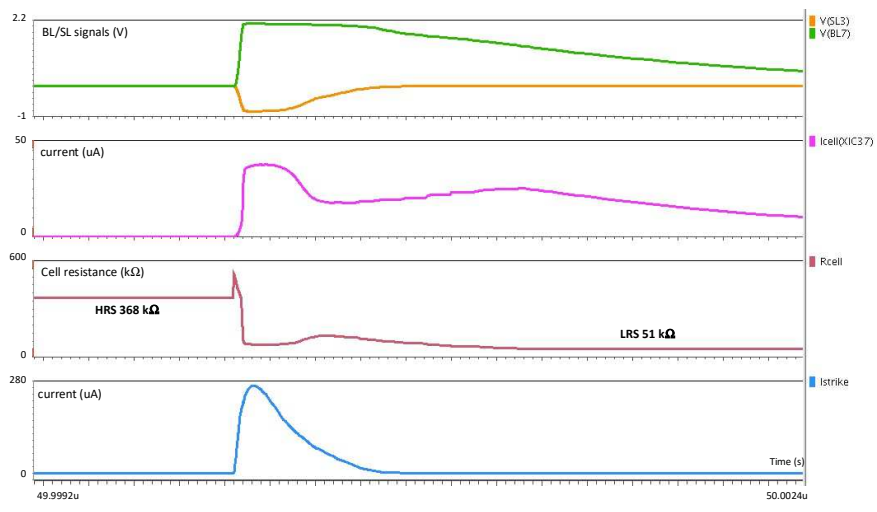

Fig. 10. Transient simulation results for a particle Al hor. striking a cell in HRS state

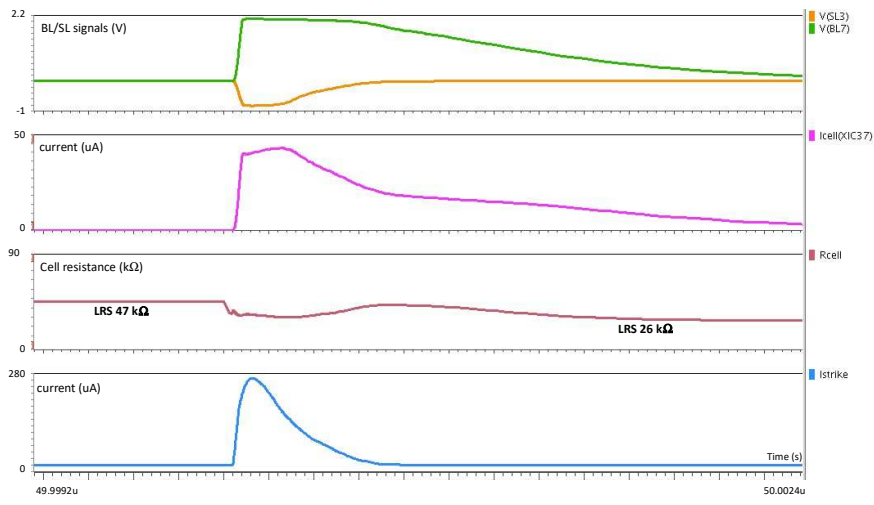

Fig. 11. Transient simulation results for a particle Al hor. striking a cell in a LRS state

Fig. 10 shows the impact of a particle (at 50 us) on a HRS cell in STBY mode. The simultaneous impact of the particle on BL and SL nodes induces a voltage difference across the cell of the memory array connected to the impacted drivers. Then, a programming current through the cell is triggered and a change of the cell resistance from HRS to LRS is detected. This event is named Single Event Upset (SEU) as the memory state is switched with respect to the OxRAM nominal values. For each simulated configuration in this work, a PMOS is simultaneously impacted with a NMOS. Then a negative current is injected when a NMOS is supposed to be struck, and a positive current for a PMOS.

Fig. 11 shows the impact of the Al hor. particle on a LRS cell in STBY mode. In this configuration, a shift of memory cell resistance towards lower LRS levels is observed. This resistance shift improves the LRS. However, going deeper in the LRS state can lead to programming issues during the next RESET operation. Indeed, if the LRS resistance value is too low, resetting the cell to HRS can be difficult or even impossible [18]. Then a stuck at fault will occur.
Table III summarizes the particle impacts on the cell resistance when the cell is in an initial HRS or LRS state. Resistance shift, compared to the resistance nominal value before the strike, or radiative event (SEU or stuck-at-fault) are captured and presented. The analysis was conducted for the different current sources correlated with Alpha and Aluminum particles and presented in Section III. From this table, we can conclude that the SEU will occur only in the HRS state and for a particle striking simultaneously a NMOS connected to the BL node and a PMOS connected to the SL node. The opposite configuration does not induce errors but resistance shifts. Concerning the initially LRS state, for each configuration the LRS state is reinforced, potentially leading to stuck-at-faults depending on the value of the resistance shift (Stuck?). The resistance shift is then related to the injected current and the occurrence of stuck-atfaults would depend on the threshold of the RRAM cell. The resistance shift seems to be linked to the peak value of the generated currents. From Tab. III and from Fig. 3, we can note that the resistance shift is $28 \%$ for the Alpha particles, $39 \%$ for the Aluminum in the diagonal configuration and $45 \%$ for the Aluminum in the horizontal configuration. This clearly follows the trend of the current peaks presented in Fig. 3. This peak is linked to the deposited energy. Then the stuck-at-fault occurrence would depend on the energy deposited by the particle in the sensitive transistors surrounding the RRAM.

TABLE III. WORST CASE SCENARIOS RESULTS IN STBY MODE

\begin{tabular}{|c|c|c|c|c|c|}
\hline & State & $\begin{array}{r}\text { Negative } \\
\text { current }\end{array}$ & $\begin{array}{c}\text { Positive } \\
\text { current }\end{array}$ & $\begin{array}{c}\text { Resistance } \\
\text { shift (\%) }\end{array}$ & Effect \\
\hline \multirow{4}{*}{$\begin{array}{l}\text { He diag. } \\
\text { He hor. } \\
\text { Al diag. } \\
\text { Al hor. }\end{array}$} & HRS & $\mathrm{BL}$ & SL & -80 & SEU \\
\hline & HRS & $\mathrm{BL}$ & SL & -80 & SEU \\
\hline & HRS & $\mathrm{BL}$ & SL & -85 & SEU \\
\hline & HRS & $\mathrm{BL}$ & SL & -86 & SEU \\
\hline \multirow{4}{*}{$\begin{array}{l}\text { He diag. } \\
\text { He hor. } \\
\text { Al diag. } \\
\text { Al hor. }\end{array}$} & LRS & $\mathrm{BL}$ & SL & -28 & Stuck? \\
\hline & LRS & $\mathrm{BL}$ & $S L$ & -28 & Stuck? \\
\hline & LRS & $\mathrm{BL}$ & SL & -39 & Stuck \\
\hline & LRS & $\mathrm{BL}$ & SL & -45 & Stuck \\
\hline \multirow{4}{*}{$\begin{array}{l}\text { He diag. } \\
\text { He hor. } \\
\text { Al diag. } \\
\text { Al hor. }\end{array}$} & HRS & SL & $\mathrm{BL}$ & 0 & NO \\
\hline & HRS & SL & $\mathrm{BL}$ & 0 & NO \\
\hline & HRS & $S L$ & $\mathrm{BL}$ & 0 & NO \\
\hline & HRS & SL & $\mathrm{BL}$ & 0 & NO \\
\hline \multirow{4}{*}{$\begin{array}{l}\text { He diag. } \\
\text { He hor. } \\
\text { Al diag. } \\
\text { Al hor. }\end{array}$} & LRS & $S L$ & $\mathrm{BL}$ & -28 & Stuck? \\
\hline & LRS & SL & $\mathrm{BL}$ & -28 & Stuck? \\
\hline & LRS & SL & $\mathrm{BL}$ & -39 & Stuck \\
\hline & LRS & SL & $\mathrm{BL}$ & -45 & Stuck \\
\hline
\end{tabular}

\section{CONCLUSION}

This paper provides a complementary analysis of Single Event Effect in a RRAM memory cell. As it was shown in [17], some specific configurations can induce effects in this kind of device, particularly when striking simultaneously a NMOS and a PMOS transistors, in standby operation. The configurations simulated here show that SEU could occur in RRAM cells for some specific topologies. This is the case if a NMOS connected to $\mathrm{BL}$ node is close enough to the PMOS connected to the SL node to be crossed by a single particle when initially at HRS. By 
avoiding this topology, SEU could be reduced. Other configurations, related to the LRS, can induce stuck-at-fault which could be more difficult to address than SEU because the cell then remains permanently in LRS.

Finally, the study was performed on a $130 \mathrm{~nm}$ technology. At the transistor level, downscaling should induce a shrinking of the drain/source junctions and should make them closer. As transistors for smaller technology nodes are known to be more sensitive to ionizing particles, the SEE rate should increase. Moreover, at the memory matrix level, the shrunk transistors should be closer, facilitating the crossing of a single particle through several sensitive transistors and making the scenario studied in this paper more probable.

\section{REFERENCES}

[1] I.G. Baek et al., "Multi-layer cross-point binary oxide resistive memory (OxRAM) for post-NAND storage application", IEDM Tech. Dig., pp. 750-753, 2005.

[2] B. Butcher, X. He, M. Huang, Y. Wang, Q. Liu, H. Lv, M. Liu, WeiWang, "Proton-based total-dose irradiation effects on $\mathrm{Cu} / \mathrm{HfO} 2: \mathrm{Cu} / \mathrm{Pt}$ RRAM devices", IOP Nanotechnology, October 29, 2010.

[3] H. Aziza, M. Bocquet, J.-M. Portal, C. Muller, "Bipolar OxRAM memory array reliability evaluation based on fault injection", IDT 2011, 2011

[4] M. Bocquet, D. Deleruyelle, C. Muller, J.-M. Portal, "Self-consistent physical modeling of set/reset operations in unipolar resistive-switching memories", Appl. Phys. Lett., vol. 98, no. 26, pp. 263507(1-3), 2011.

[5] S. Gerardin and A. Paccagnella, "Present and Future Non-Volatile Memories for Space", IEEE Trans.On Nucl. Sc., pp. 3016-3039, Vol. 57, No. 6, Dec. 2010.

[6] J. Bi and Yuan Duan and K. Xi and B. Li, "Total ionizing dose and single event effects of $1 \mathrm{Mb}$ HfO2-based resistive-random-access memory“, Microelectron. Reliab.\}, 2018\}, volume 88-90, pages 891-897

[7] Y. Wang et al., "The heavy ion radiation effects on the $\mathrm{Pt} / \mathrm{HfO} 2 / \mathrm{Ti}$ resistive switching memory," 2016 16th European Conference on Radiation and Its Effects on Components and Systems (RADECS), 2016, pp. 1-3, doi: 10.1109/RADECS.2016.8093118.

[8] A. M. S. Tosson, S. Yu, M. H. Anis and L. Wei, "1T2R: A novel memory cell design to resolve single-event upset in RRAM arrays," 2017 IEEE 12th International Conference on ASIC (ASICON), 2017, pp. 12-15, doi: 10.1109/ASICON.2017.8252399.

[9] Yan Wang, Hangbing Lv, Wei Wang, Qi Liu, Shibing Long, Qin Wang, Zongliang Huo, Sen Zhang, Yingtao Li, Qingyun Zuo, Wentai Lian, Jianhong Yang, and Ming Liu, "Highly Stable Radiation-Hardened Resistive-Switching Memory", IEEE Elec. Dev. Let., pp 1470-1472, Vol. 31, No. 12, Dec. 2010

[10] J.S.Biet al., "The impact of X-ray and proton irradiation on HfO2/Hfbased bipolar resistive memories,'IEEE Trans. Nucl. Sci., vol. 60, no. 6,pp. 4540-4546, Dec. 2013.

[11] X. He, W. Wang, B. Butcher, S. Tanachutiwat, and R. E. Geer, "SuperiorTID hardness in TiN/HfO2/TiN RRAMs after proton radiation,'IEEETrans. Nucl. Sci., vol. 59, no. 5, pp. 2550-2555, Oct. 2012.

[12] S. L. Weeden-Wrightet al., "TID and displacement damage resilienceof 1T1R HfO2/Hf resistive memories,"IEEE Trans. Nucl. Sci., vol. 61,no. 6, pp. 2972-2978, Dec. 2014.

[13] F. Yuan, S. Shen, and Z. Zhang, "V-ray irradiation effects onTiN/HfOx/Pt resistive random access memory devices," inProc. IEEEAerosp. Conf., Mar. 2015, pp. 3560-3567.

[14] Fanget al., "Total ionizing dose effect ofy-ray radiation onthe switching characteristics and filament stability of HfOxresistiverandom access memory,”Appl. Phys. Lett., vol. 104, no. 18, May 2014,Art. no. 183507.

[15] S. Petzold et al., "Heavy Ion Radiation Effects on HafniumOxide-Based Resistive RandomAccess Memory”, IEEE Trans. Nucl. Sci., vol. 66, no. 7, July. 2019
[16] K. Castellani-Coulié, M. Bocquet, H. Aziza, J-M. Portal, W. Rahajandraibe, C. Muller, "Analysis of Single Event Effects in an OxRRAM cell by using SPICE level simulations", Radiation and its Effects on Components and Systems (RADECS) 2012, Biarritz (France), 24-28 septembre 2012.

[17] K. Castellani-Coulié, M. Bocquet, H. Aziza, J. M. Portal, W. Rahajandraibe and C. Muller, "SPICE level analysis of Single Event Effects in an OxRRAM cell," 2013 14th Latin American Test Workshop LATW, 2013, pp. 1-5, doi: 10.1109/LATW.2013.6562684.

[18] M. Fieback et al., "Device-Aware Test: A New Test Approach Towards DPPB Level,’2019 IEEE International Test Conference (ITC), 2019, pp. 1-10, doi: 10.1109/ITC44170.2019.9000134.

[19] TCAD Sentaurus user's manual (Synopsys).

[20] A. Akkerman, J. Barak, D. Emfietzoglou, "Ion and electron trackstructure and its effects in silicon: model and calculation", Nuclear Instruments and Methods in Physics Research, pp. 319-336, 2005.

[21] www.srim.org

[22] Vasiliy V. Markelov and Michael G. Tverskoy, "Evaluation of LET Spectra Produced by High Energy Protons in Si", RADECS 2015, Moscou, Russia, September 2015. DOI: 10.1109/RADECS.2015.7365592

[23] A. Peczalski et al, "Physical SEU Model for Circuit Simulation", IEEE TNS, Vol. 35, No. 6, December 1988.

[24] V. Pouget et al, "SPICE Modeling of the Transient Response of Irradiated MOSFETS", IEEE TNS, Vol. 47, No. 3, June 2000.

[25] A. Douin et al, "Influence of Laser Pulse Duration in Single Event Upset Testing”, IEEE TNS, Vol. 53, No. 4, August 2006.

[26] C. Godlewski et al, "Electrical modeling of the effect of beam profile for pulsed laser fault injection", Micro. Reliability, 2009.

[27] D.E. Fulkerson et al, "Prediction of SOI Single-Event Effect Using a Simple Physics-Based SPICE Model", IEEE TNS, Dec. 2005.

[28] K. Castellani-Coulie, G. Toure, J. Portal, O. Ginez, H. Aziza and A. Lesea, "Circuit Effect on Collection Mechanisms Involved in Single Event Phenomena: Application to the Response of a NMOS Transistor in a $90 \mathrm{~nm}$ SRAM Cell," in IEEE Transactions on Nuclear Science, vol. 58, no. 3, pp. 870-876, June 2011, doi: 10.1109/TNS.2011.2129575.

[29] K. Castellani-Coulié, J. Portal, G. Micolau and H. Aziza, "Analysis of SEU parameters for the study of SRAM cells reliability under radiation," 2011 12th Latin American Test Workshop (LATW), 2011, pp. 1-5, doi: 10.1109/LATW.2011.5985895

[30] K. Castellani-Coulié, H. Aziza, W. Rahajandraibe, G. Micolau and J. -. Portal, "Development of a CMOS Oscillator Concept for Particle Detection and Tracking," in IEEE Transactions on Nuclear Science, vol. 60 , no. 4, pp. 2450-2455, Aug. 2013, doi: 10.1109/TNS.2013.2254723. 